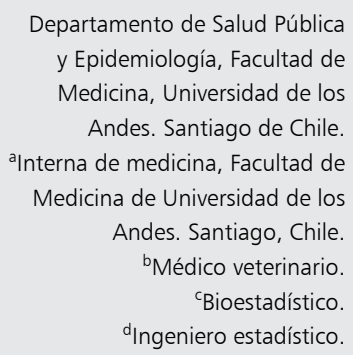

Recibido el 24 de septiembre de 2015, aceptado el 29 de marzo de 2016.

Correspondencia a: Dra. María Teresa Valenzuela Vicedecana Investigación y Postgrado.

Facultad de Medicina, Universidad de los Andes Av. Mons. Álvaro del Portillo 12.455. Las Condes, Santiago, Chile.

Teléfono: 226181806 mtvalenzuela@uandes.cl

\section{Tendencia de la tasa global de fecundidad y el aumento de la fuerza laboral femenina en Chile. 1960-2011}

\author{
MARÍA DE LOS ÁNGELES STEFANELLIa, \\ MARÍA TERESA VALENZUELA, MARCELA CÁRCAMO ${ }^{b}$, \\ CINTHYA URQUIDI, GABRIEL CAVADAc ${ }^{c}$, PAMELA SAN MARTÍN P. ${ }^{d}$
}

\section{Association between the decline in global fertility rate and the incorporation of women to the workforce}

Background: The global fertility rate (GFR) is defined as the mean number of children that a woman could have in a hypothetical cohort, not exposed to death during the fertile period. GFR has fallen from 3.4 to 1.9 children per women in the period 1970-2010. Aim: To explore the relationship between the fall in GFR and the incorporation of women to work in the period 1960-2011. Material and Methods: Data from the National Statistics Institute was used. GRF was calculated using specific fertility rates for each year considering women aged 15 to 49 years. Work rates were obtained from yearly vital statistics reports. Results: Between 1960 and 2011, GRF decreased from 5.5 to 1.9 in Chile. The first inflection occurred in 1970. In the same period, female workforce increased from 22.4 to $40.2 \%$. Conclusions: To motivate the participation of female workforce without decrease the GRF allowing population replacement, it is suggested the need to create new public policies with benefits and support from the state.

(Rev Med Chile 2016; 144: 658-663)

Key words: Demography; Fertility; Manpower; Population.

\section{L} a tasa global de fecundidad (TGF) se define, según la Organización Panamericana de la Salud (OPS) en conjunto con la Organización Mundial de la Salud (OMS), como "el número de hijos que en promedio tendría cada mujer de una cohorte hipotética de mujeres, no expuesta al riesgo de muerte a lo largo del período fértil y que experimentase las tasas de fecundidad específicas por edad prevalentes en un determinado año o periodo, para un determinado país, territorio o área geográfica"'.

Al comenzar el siglo XXI, prácticamente en todos los países desarrollados, incluyendo la mayor parte de Europa, el este de Asia y muchos de los países americanos -desde Canadá hasta Chile- la tasa de fecundidad ha descendido por debajo de los niveles necesarios, generando así el envejecimiento acelerado de la población y su deterioro. Esta situación, asociada al aumento de la esperanza de vida, han generado un importante impacto en las características demográficas de nuestra población, con una distinta conformación de grupos etarios, donde se observa un incremento de adultos mayores, sin compensación de los grupos etarios menores, generando implicancias tanto en políticas de salud pública como en las económicas y sociales ${ }^{2}$.

Actualmente, en promedio, las mujeres en un país desarrollado tienen sólo 1,66 hijos a lo largo de su vida, lo que las sitúa alrededor de $35 \%$ por debajo de la cifra necesaria para garantizar el recambio generacional, siendo este, el índice de 
fecundidad necesario para asegurar la sustitución de una generación por otra de igual tamaño $(2,1$ hijos por mujer) y mantener la población a largo plazo. Esto supone que el número de niños de entre 0 y 14 años en el mundo desarrollado es 60,6 millones menor en la actualidad que en el año 1965 y debido, precisamente, a la escasez de niños, los países desarrollados se encuentran ante una tasa de mano de obra decreciente, y que deben enfrentarse al reto de ayudar a una población anciana que crece rápidamente ${ }^{3}$.

En parte, esta drástica disminución se explica por el aumento de la fuerza laboral femenina (FLF), donde las mujeres han logrado mayores oportunidades económicas en las sociedades avanzadas, entendiéndose ésta como la población femenina de 15 años y más, que suministra la mano de obra para la producción de bienes y servicios, esto es, ocupados y desocupados ${ }^{4}$.

Las mujeres jóvenes y con estudios, por lo general, no tienen necesidad económica ni interés de abandonar o comprometer el desarrollo de sus carreras profesionales para convertirse en madres ${ }^{3}$. La disminución de los salarios y el incremento de la inseguridad, en cuanto a la estabilidad en el puesto de trabajo, han jugado un papel importante para muchas parejas jóvenes, llevándolas a la conclusión de que deberían permanecer sin hijos o retrasar el matrimonio y construir una familia ${ }^{5}$.

La Organización de las Naciones Unidas (ONU) pronostica que para el 2035, la población de niños menores de 5 años, ya en declive en muchos de los países desarrollados, caerá también a nivel global ${ }^{6}$. Esto significa que la cifra de población mundial podría estar descendiendo con el cambio de siglo, sobre todo si las tasas de fecundidad siguen con tendencia a la baja.

En Chile, la TGF ha caído notablemente en los últimos cuarenta años ${ }^{7}$, desde 3,4 hasta 1,9 niños por mujer en el período de 1970 a 2010. Parte de este declive, al igual que en el resto del mundo, se debe al creciente desarrollo y a la urbanización, que hacen que tener hijos sea más caro en el corto plazo $^{6}$. Entre otros posibles factores se incluyen el declive de la participación religiosa, el auge del individualismo ${ }^{8}$, así como el aumento de la participación de la mujer en el mundo laboral y la dificultad que tienen algunas mujeres chilenas de conciliar su vida familiar con su vida laboral ${ }^{9}$. El objetivo de este estudio es evaluar la relación existente entre el descenso de la TGF en Chile y la incorporación de la FLF entre los años 1960 y 2011 $y$, de acuerdo a ello, incentivar a los tomadores de decisiones para plantear estrategias que permitan garantizar el recambio generacional.

\section{Material y Método}

Estudio ecológico de series de tiempo cuyo período objetivo fue entre los años 1960 y 2011.

La TGF fue obtenida a través del cálculo de tasas específicas de fecundidad para cada año censal, considerando a las mujeres en edad fértil -15 a 49 años de edad- de Chile.

Para la obtención de los datos se usó la información censal desde 1960 a 2002 y para los años 2002 a 2011, se utilizaron los datos registrados en los informes anuales de estadísticas vitales, elaborados por el Instituto Nacional de Estadísticas $(\mathrm{INE})^{10,11}$

La FLF fue calculada sólo considerando cantidad de mujeres ocupadas mayores de 15 años, datos obtenidos desde los informes censales y estadísticas anuales de fuerza laboral, elaborados por el INE $\mathrm{IN}^{10-13}$.

Los análisis de tendencia y asociación de la TGF, con las variables tiempo y FLF, se realizaron a través del modelo lineal autoregresivo de primer orden (Regresión de Prais-Winsten) ${ }^{14}$, usando el software estadístico Stata versión 13.0, con nivel de significación de $5 \%$.

\section{Resultados}

La TGF ha experimentado un sostenido descenso en Chile, disminuyendo desde 5,49 a 1,86 entre los años 1960 y 2011, respectivamente.

La primera inflexión de tendencia ocurrió en la década 1970-79, donde la TGF bajó a 2,67. Es así como en la mitad de la primera década del siglo XXI, este indicador alcanzó el valor más bajo del período analizado, 1,8 hijos por mujer.

La incorporación de la mujer a la fuerza laboral ha presentado un incremento sostenido, variando desde $22,36 \%$ a 40,21\% entre los años 1960 y 2011, respectivamente (Tabla 1) (Figuras 1 y 2 ).

Se constató una relación exponencial entre la TGF y el porcentaje de FLF. Este modelo explica el $84 \%$ de la variabilidad $(\mathrm{p}=0,000)$. La expre- 
Tabla 1. Evolución de la TGF y FLF en Chile, año 1960 a 2011

\begin{tabular}{|ccc|}
\hline Año & TGF & FLF (\%) \\
\hline 1960 & 5,49 & 22,36 \\
1970 & 4,44 & 24,15 \\
\hline 1982 & 2,67 & 26,07 \\
\hline 1992 & 2,53 & 29,61 \\
\hline 2002 & 1,98 & 34,86 \\
\hline 2003 & 1,92 & 33,40 \\
\hline 2004 & 1,87 & 34,26 \\
\hline 2005 & 1,82 & 35,30 \\
\hline 2006 & 1,80 & 35,70 \\
\hline 2007 & 1,86 & 35,88 \\
\hline 2008 & 1,89 & 37,18 \\
\hline 2009 & 1,92 & 37,45 \\
\hline 2010 & 1,90 & 38,06 \\
\hline 2011 & 1,86 & 40,21 \\
\hline
\end{tabular}

sión analítica de esta relación está contenida en la siguiente ecuación:

$$
T G F=16,28 \operatorname{Exp}^{(-0,059) *}(\% F L F)
$$

Se muestra en la Figura 3.

Al evaluar las ecuaciones en una TGF igual a 2 (tasa mínima de reemplazo demográfico), se encuentra que el porcentaje de FLF es de aproximadamente $36 \%$ para el periodo estudiado.

\section{Discusión}

Este estudio logró establecer la importante relación que existe entre la TGF y la participación femenina en la fuerza de trabajo en Chile.

Si bien se desconoce el porcentaje exacto actual de la fuerza de trabajo femenina, se sabe por los

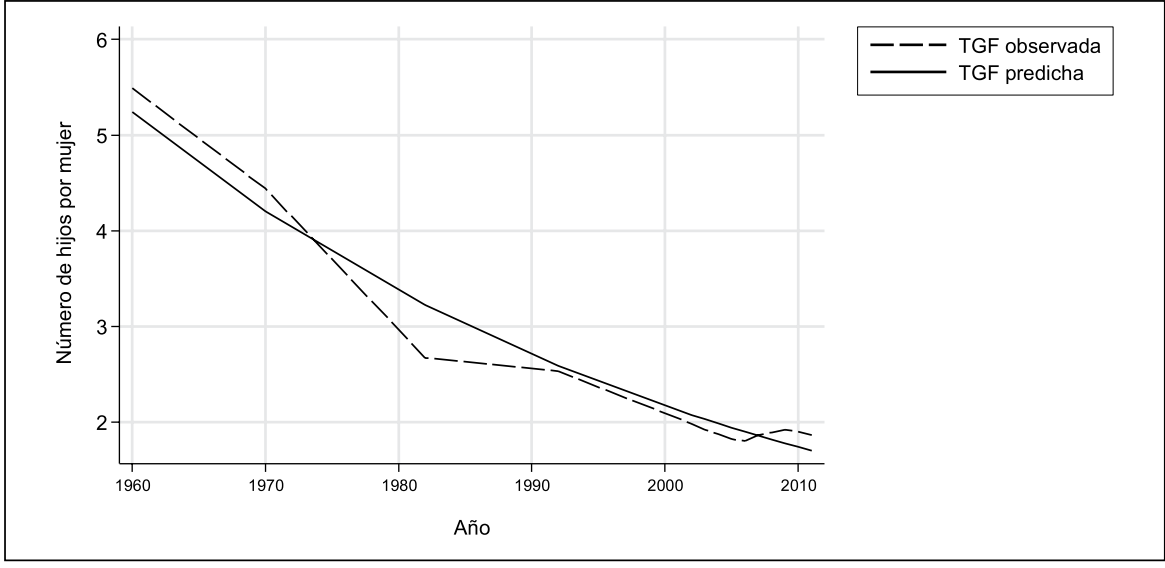

Figura 1. TGF observada y predicha. Chile 1960-2011.

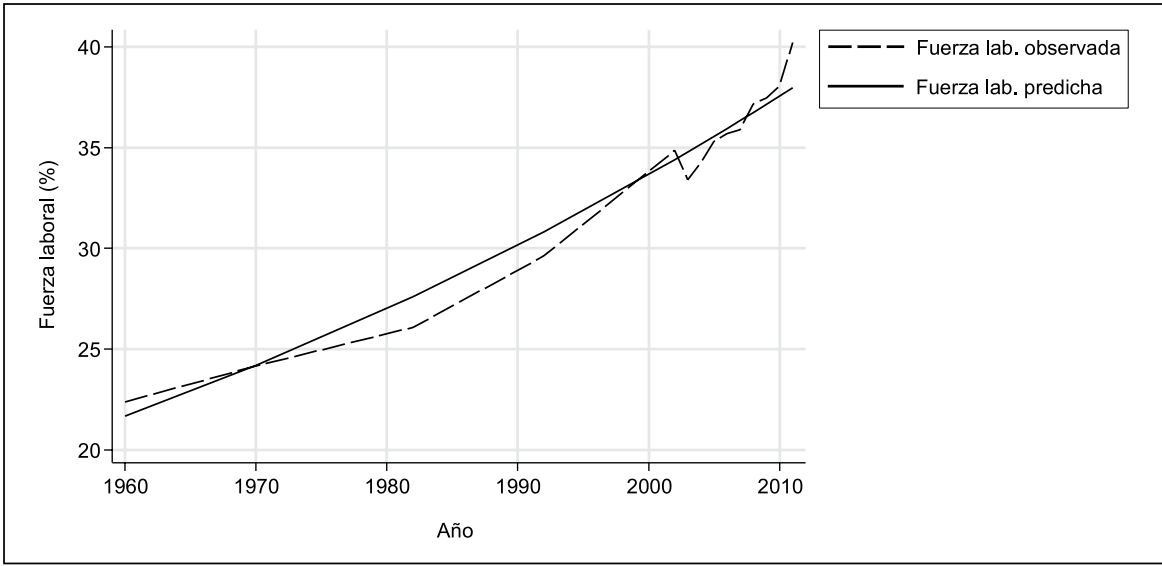

Figura 2. FLF (\%) observada y predicha. Chile 1960-2011. 


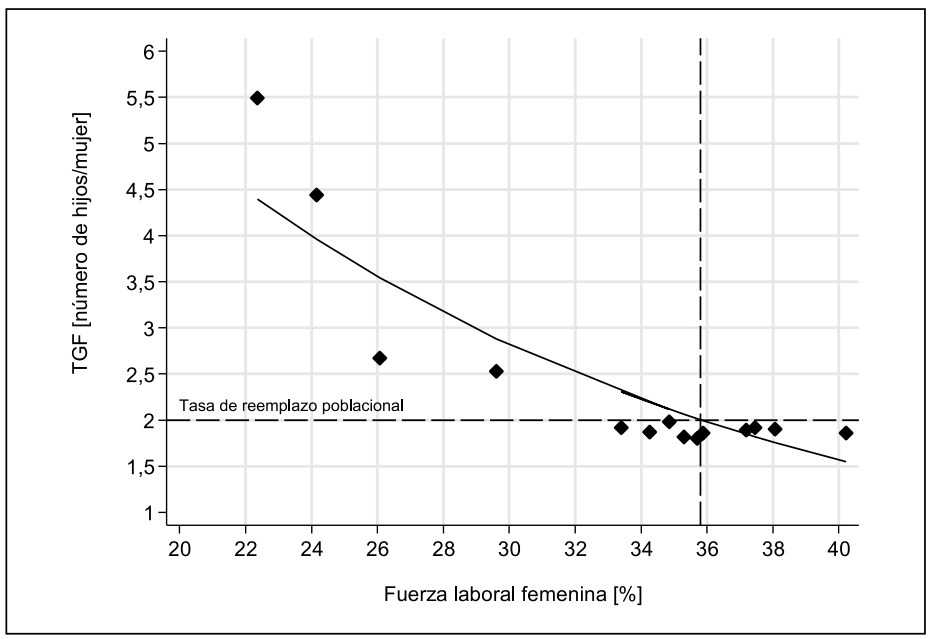

Figura 3. FLF y TGF. Chile 1960-2011.

datos recolectados, que sobrepasa el $40 \%{ }^{12,13}$. Con esta información, lejos de pretender cercenar el acceso al trabajo de las mujeres chilenas, lo que se pretende es incentivar la promulgación de nuevas políticas públicas que permitan compatibilizar el trabajo y la maternidad, ambas, actividades y vivencias, que toda mujer tiene derecho de elegir.

La tendencia a la reducción de la tasa de fecundidad se debe a múltiples factores, los cuales no son objetivo de este trabajo, sin embargo, deben ser considerados a la hora de generar políticas públicas. Dentro de las causas principales cabe mencionar el uso de métodos anticonceptivos y otros medios de regulación de la fertilidad, introducidos en Chile a fines de la década 1960$69^{15,16}$; en segundo lugar cobra gran importancia el cambio de los roles femeninos, quienes han adquirido un mayor nivel educativo, teniendo grandes oportunidades de trabajo y de mayores ingresos fuera de casa, lo que asimismo lleva a la utilización de medios de anticoncepción, por la intrincada tarea que supone desenvolverse entre el trabajo, sus exigencias, horarios y el cuidado de un niño.

Además, el auge de la urbanización ha generado un aumento del costo de la crianza de los hijos. El costo de la educación es alto y no sólo eso, sino el costo asociado a la vida urbana también es considerable. Las necesidades se han ido masificando también, algunas de ellas son esenciales y otras más superfluas. Por último, reconocer los cambios culturales y creencias religiosas que finalmente moldean las sociedades en cada época de la historia ${ }^{3,17}$.

Parece pertinente entonces, estudiar las conductas que otros países en la misma situación han tomado. El gran ejemplo de ello es Europa, donde la TGF en todos los países miembros de la Unión Europea es menor de 2, generando un muy lento crecimiento poblacional o en definitiva una disminución de la población ${ }^{18}$. La disminución de la fecundidad, junto al aumento de la esperanza de vida, origina un aumento del grupo de adultos mayores y, en el caso de Europa, se estima que para el 2050 la población mayor de 65 años será de $30 \%{ }^{18}$. Esto ensombrece el futuro económico de las naciones del viejo continente, pues disminuye la fuerza trabajadora, disminuye la productividad y entorpece la solvencia de pensiones y de seguros sociales, poniendo grandes barreras para alcanzar metas como el empleo pleno, crecimiento económico y cohesión social.

Por ejemplo, Francia era uno de los países europeos con una de las TGF más bajas en 1970 y luego que el gobierno dispusiera de un plan fomentador tanto de la fertilidad como de la convivencia entre trabajo y cuidado de hijos, ha logrado convertirse en el segundo país de Europa con mayor TGF, presentando inclusive un aumento entre 1993 y $2002^{18}$. Otro ejemplo es Suecia, que ha creado horarios flexibles de trabajo, una red de centros de cuidado de niños de calidad y un posnatal prolongado a veces de hasta 450 días, con términos económicamente razonables ${ }^{19}$. 
Un aspecto relevante a considerar al momento de ejecutar políticas de salud pública, más aun si involucra fertilidad, es el contexto económico de cada país. Es distinto efectuar medidas en una nación desarrollada y estable económicamente, con ingresos PIB per cápita de US $\$ 60.380,4$ como Suecia, US\$46.251,4 Alemania o de US\$42.560,4 como Francia; que implementarlas en un país en vías de desarrollo como Chile, que posee un ingreso per cápita de US $\$ 15.732,3^{20}$.

Por lo expuesto, la sociedad chilena debe sin demora, comenzar a formular estrategias ante la gran tarea que enfrenta. Las nuevas políticas deben apuntar a posicionar aun mejor a las mujeres en el mundo laboral y a aspirar que ellas reciban altos niveles de educación; es más, gracias a estos logros se ha impactado favorablemente la reducción de la mortalidad infantil ${ }^{21-23}$. Alargar el posnatal, que éste tenga una remuneración adecuada, generación de un incentivo a la maternidad, prolongándolo hasta la incorporación de los hijos a la educación básica, adaptar horarios de trabajo y que existan guarderías que ofrezcan un servicio de calidad, entre varias otras.

Como aspecto importante, considerar la creación de una compensación equivalente a las ganancias familiares que se estarían viendo disminuidas al postergar o interrumpir el ingreso de la fuerza laboral de la mujer.

A muchos esto les parecerá poco atractivo, pues supone un gasto considerable de dinero, cuyos resultados se observarán a largo plazo, en tiempos de una nueva generación. Muchos optarán por ignorar el tema o instaurar medidas que sólo alargan el inicio de soluciones reales, como lo es aceptar inmigrantes sólo por lo que representan como fuerza de trabajo. Sin embargo, para aquellos reticentes a ahondar en este importantísimo conflicto, es bueno preguntarse: ¿Estoy dispuesto a jubilar 5 años más tarde porque no hay población productiva en mi país? O para los que piensan que es un expendio que el Estado no puede pagar, ¿cuánto dispone el Estado para solventar las licencias médicas a menores de un año en Chile? Probablemente no sea menos dinero del que se gastaría implementando medidas que fomenten la maternidad.

Este último es un tema interesante para investigar en un siguiente estudio, con el cual se podría avaluar el gasto necesario para alcanzar una tasa global de fecundidad adecuada.

\section{Conclusión}

Existe relación exponencial entre la TGF y el porcentaje de FLF.Se sugiere la necesidad de generar nuevas políticas referentes a incentivar a las madres a concebir más hijos, con los respectivos respaldos del Estado.

\section{Referencias}

1. Organización Panamericana de la Salud: Unidad de Información y análisis de Salud (HA). Iniciativa Regional de Datos Básicos en Salud: Glosario de Indicadores [en línea] [consultado el 19 de junio de 2014]. Washington DC, 2013. Disponible en: http://www.paho.org/hq/ index.php?option $=$ com_docman \&task=doc_download\&gid=23508\&Itemid=270\&lang=es.

2. Martínez R, Ranero V, Vega E. Crecimiento acelerado de la población adulta de 60 años y más de edad: Reto para la salud pública [en línea] [consultado el 26 de abril de 2014]. Washington DC, 2012. Disponible en: http:// www.paho.org/hq/index.php?option=com_content\&view $=$ article\&id $=2796 \% 3$ Acrecimiento-acelerado-poblacion-adulta-60-anos-mas-edad-reto-salud-publica\&catid $=1796 \% 3$ Afacts $\&$ Itemid $=\&$ lang $=p t$

3. Social Trendial Institute. El dividendo demográfico sostenible. ¿Qué tienen que ver el matrimonio y la fecundidad con la economía? [en línea] [consultado el 28 de abril de 2015]. New York 2012. Disponible en: http:// www.socialtrendsinstitute.org/upload/publications/ family/DividendoDemograficoSostenible.pdf

4. Ministerio del Desarrollo Social. Gobierno de Chile: Observatorio Social-Encuesta Casen: Definiciones e indicadores [en línea] [consultado el 28 de abril de 2015]. Santiago, 2013. Disponible en: http://observatorio.ministeriodesarrollosocial.gob.cl/casen/casen_def_trabajo. php

5. Naciones Unidas. CEPAL. División de Desarrollo Social. UNICEF: La evolución de las estructuras familiares en América Latina, 1990-2010. Los retos de la pobreza, la vulnerabilidad y el cuidado [en línea] [consultado el 28 de mayo de 2015]. Santiago, 2014. Disponible en: http://www.cepal.org/es/publicaciones/36717-la-evolucion-de-las-estructuras-familiares-en-america-latina-1990-2010-los-retos.

6. Bryant J. Theories of Fertility Decline and the Evidence from Development Indicators. Population Development Review 2007; 33 (1): 101-27.

7. Vera X. ¿Hacia o en la segunda transición demográfica? Los cambios poblacionales de Chile desde un enfoque de 
género. Revista Anales de la Universidad de Chile 2012; 105-25.

8. Lehmann C. ¿Chile un país católico? Puntos de Referencia 249: Centro de Estudios Públicos [en línea] [consultado el 28 de abril de 2015]. Santiago 2001. Disponible en: http://www.cepchile.cl/dms/archivo_2980_472/ pder249_lehmann.pdf

9. United Nations. Department of Economic and Social Affairs. Population Division: Population Bulletin of the United Nations. Completing the fertility transition [en línea] [consultado el 28 de abril de 2015]. New York, 2009. Disponible en: http://www.un.org/esa/population/ publications/completingfertility/bulletin-english.pdf

10. Instituto Nacional de Estadísticas. Gobierno de Chile. Estadísticas Vitales del 2002 al 2012 [en línea] [consultado el 18 de junio de 2014]. Santiago, 2014. Disponible en: http://www.ine.cl/canales/chile_estadistico/demografia_y_vitales/estadisticas_vitales/estadisticas_vitales. php

11. Instituto Nacional de Estadísticas. Gobierno de Chile. Censos históricos [en línea] [consultado el 18 de junio de 2014]. Santiago, 2003. Disponible en: http://www.ine. cl/canales/chile_estadistico/censos/censo_poblacion_vivienda.php

12. Banco Central de Chile. Bases de Datos Estadísticos. Mercado Laboral y Demografía [en línea] [consultado el 18 de junio de 2014]. Santiago, 2014. Disponible en: http://si3.bcentral.cl/Siete/secure/cuadros/arboles.aspx

13. Banco Central de Chile. Departamento Publicaciones de la Gerencia de Investigación Económica División Estudios. Indicadores Económicos y Sociales de Chile 19602000 [en línea] [consultado el 18 de junio de 2014]. Santiago, 2001. Disponible en: http://www.bcentral.cl/ publicaciones/estadisticas/informacion-integrada/pdf/ indicadores 19602000.pdf

14. Stata Technical Support. A publication to promote communication among Stata users [en línea] [consultado el 18 de junio de 2014]. New York, 2005. Disponible en: http://www.stata-press.com/journals/stbcontents/stb25. pdf

15. Ministerio de Salud. Gobierno de Chile. Normas Nacionales sobre Regulación de la Fertilidad: Normas técnicas y guías clínicas sobre regulación de la fertilidad [en línea] [consultado el 18 de junio de 2014]. Santiago, 2006. Disponible en: http://web.minsal.cl/portal/url/ item/795c63caff4ede9fe04001011f014bf2.pdf

16. Donoso E, Carvajal J, Domínguez M. Reducción de la fecundidad y envejecimiento de la población de mujeres chilenas en edad fértil: 1990-2004. Rev Med Chile 2009; 137: 766-73.

17. Ministerio Secretaría General de la Presidencia. Gobierno de Chile. Informe de las Comisiones Asesoras Presidenciales 2010-2014 [en línea] [consultado el 28 de abril de 2015]. Santiago, 2014. Disponible en: http:// gestion2010-2014.cumplimiento.gob.cl/wp-content/ uploads/2014/03/140319_informes_comisiones_asesoras_presidenciales_2010-2014.pdf

18. RAND Europe-Cambridge. Population Implosion? Low Fertility and Policy Responses in the European Union [en línea] [consultado el 18 de junio de 2014]. Cambridge, 2005. Disponible en: http://www.rand.org/content/ dam/rand/pubs/research_briefs/2005/RAND_RB9126. pdf

19. Zárate A. Un estudio de las ayudas directas a la natalidad como instrumento para incentivar la fecundidad y luchar contra la despoblación. Departamento de Estructura e Historia Económica y Economía Pública Universidad de Zaragoza [en línea] [consultado el 18 de junio de 2014]. Zaragoza, 2002. Disponible en: http://www. ceddar.org/content/files/articulof_272_01_DT2002-2. pdf

20. Banco Mundial. PIB per cápita (US\$ a precios actuales) [en línea] [consultado el 26 de mayo de 2015]. Washington, 2015. Disponible en: http://datos.bancomundial. org/indicador/NY.GDP.MKTP.CD

21. Kaempffer A, Medina E. Mortalidad infantil reciente en Chile: Éxitos y desafíos. Rev Chil Pediatr 2006; 77 (5): 492-500.

22. Barría M, Venegas J. Aproximaciones metodológicas al estudio de la mortalidad infantil en Chile. Rev Chil Pediatr 2012; 83 (1): 33-41.

23. González R. Chile un buen lugar donde nacer: morbimortalidad materna e infantil a nivel global y nacional. Rev Med Clin Condes 2014; 25 (6): 874-8. 